\title{
ESTUDIO DESCRIPTIVO DEL USO DE DROGAS EN ADOLESCENTES DE EDUCACIÓN MEDIA SUPERIOR DE LA CIUDAD DE MONTERREY, NUEVA LEÓN, MÉXICO
}

Gregorio Martinez Rodríguez 2 Margarita Antonia Villar Luis $^{3}$

Rodríguez GM, Luis MAV. Estudio descriptivo del uso de drogas en adolescentes de educación media superior de la ciudad de Monterrey, Nueva León, México. Rev Latino-am Enfermagem 2004 março-abril; 12(número especial):391-7.

Se buscó establecer el perfil de consumo de drogas lícitas e ilícitas, patrones de consumo y estrategias de afrontamiento y resistencia al alcohol, tabaco y otras drogas en adolescentes de educación media superior. El estudio se sustentó en los conceptos de autores especializados en el tema considerándose las características conductuales, psicológicas y normativas de la persona. La muestra no probabilística consistió de 325 jóvenes. Los resultados indicaron que 67\%, 65\% y $7 \%$ de los adolescentes aceptaron haber usado alcohol, tabaco y marihuana alguna vez en su vida respectivamente y en el último mes el $33 \%, 38 \%$ y $3 \%$ esas mismas substancias, $65 \%$ consideran que la mayoría de sus compañeros han hecho uso; $56 \%$ tuvieron que rechazar una vez el ofrecimiento de alcohol, 64\% tabaco y 51\% marihuana, en el último mes con base en esos hallazgos. Se recomienda implementar un programa de prevención orientado a apoyar los recursos del adolescente para resistir las presiones para el consumo.

DESCRIPTORES: uso de drogas; estudiantes; enfermería

\section{DESCRIPTIVE STUDY OF DRUGS USE AMONG ADOLESCENTS IN HIGHER} MIDDLE EDUCATION IN MONTERREY, NUEVA LEÓN, MEXICO

We aimed to establish the consumption profile of licit and illicit drugs, consumption patterns and strategies for coping and resisting alcohol, tobacco and other drugs among adolescents in higher middle education. This study was based on concepts developed by specialized authors and considered the subject's behavioral, psychological and normative characteristics. The non-probabilistic sample consisted of 325 adolescents. Results indicated that $67 \%, 65 \%$ and $7 \%$ of the adolescents, respectively, accepted they had already used alcohol, tobacco and marihuana at some time in their life and that 33\%, 38\% and $3 \%$, respectively, had used these same substances over the last month; $65 \%$ consider most of their colleagues have used these substances; $56 \%$ had to reject an offer to consume alcohol, $64 \%$ tobacco and $51 \%$ marihuana over the last month. On the basis of these findings, we recommend the implementation of a prevention program aimed at supporting the adolescent's resources to resist pressure towards consumption.

DESCRIPTORS: drugs use; students; nursing

\section{ESTUDO DESCRITIVO DO USO DE DROGAS EM ADOLESCENTES DE EDUCACÃO MÉDIA SUPEIROR DA CIDADE DE MONTERREY, NOVA LEON, MÉXICO}

Buscou-se estabelecer o perfil de consumo de drogas lícitas e ilícitas, padrões de consumo e estratégias de enfrentamento e resistência ao álcool, tabaco e outras drogas em adolescentes de educação média superior. O estudo fundamentou-se nos conceitos de autores especializados no tema, considerando-se as características comportamentais, psicológicas normativas da pessoa. A amostra, não probabilística, consistiu de 325 jovens. Os resultados indicaram que $67 \%$, $65 \%$ e $7 \%$ dos adolescentes aceitaram haver usado álcool, tabaco e maconha alguma vez na vida respectivamente e no último mês, 33\%, 38\% e 3\% essas mesmas substâncias; 65\% consideram que a maioria de seus companheiros fez uso; $56 \%$ tiveram que recusar uma vez a oferta de álcool, 64\% tabaco e 51\% maconha, no último mês. Com base nesses achados recomendase a implementação de um programa de prevenção orientado a apoiar os aportes dos adolescentes para resistir à pressão para o consumo.

DESCRITORES: uso de drogas; estudantes; enfermagem

\footnotetext{
${ }^{1}$ Las opiniones expresadas en este articulo son de responsabilidad exclusiva de los autores y no representan la posición de la organización donde trabajan o de su administración; ${ }^{2}$ Investigador - Licenciado en enfermería, Profesor en la Universidad Autónoma de Nuevo Leon, correo electrónico: gregmarro@hotmail.com.br; ${ }^{3}$ Docente de la Escuela de Enfermería de Ribeirão Preto, de la Universidad de São Paulo, Centro Colaborador de la OMS para el desarrollo de la investigación en enfermería
} 
INTRODUCCIÓN

\section{Planteamiento del problema}

El fenómeno de las drogas se ha convertido en un problema de salud pública en los diferentes países; aún y cuando registran similitudes muy marcadas, las estrategias de control generalizado no han resultado tan eficaces por ser un problema multicausal, reflejado por el creciente aumento, la diversidad de substancias, métodos y procedimientos utilizados para el consumo en cualquier estrato social ${ }^{(1)}$.

Las estadísticas del inicio del uso de drogas se han acortado de los 15 hasta los 8 años y menos ${ }^{(2)}$. En un estudio de consumo de alcohol y tabaco en población escolar americana equivalentes al primer grado de secundaria registra un consumo de tabaco de $8,8 \%$, en el tercer grado $15,8 \%$ y para el primer año de preparatoria aumenta a $22,4 \%$, para el consumo de alcohol en las ultimas dos semanas es del $13,7 \%, 24,3 \%$ y $31,5 \%$ respectivamente, y los motivos referidos para el uso de varían desde la curiosidad, sentirse bien en relax, necesidad de ser aceptados, hacer amigos, temor, frustración, recurso para afrontar conflictos personales, y conducta aprendida de personas significativas. Los problemas escolares asociados al consumo varían desde baja escolaridad, conductas inapropiadas, delincuencia y conflictos familiares ${ }^{(3-5)}$.

Los estudios para Latinoamérica y Brasil con uso de metodología equivalente refieren una coincidencia en la mayor prevalencia de uso de drogas, de alcohol, tabaco y marihuana ${ }^{(6-7)}$ en México y predomina en otras adolescentes de 12 a 17 años el consumo del tabaco y alcohol, en las drogas ilícitas es la marihuana y cocaína y las anfetaminas las de mayor uso y duplica su grado de consumo de $1,6 \%$ a $3,2 \%$, el consumo de adolescentes que abandonan la escuela, es tres veces mayor sugiriéndose que la escuela es un factor protector ${ }^{(8)}$, y el no uso en los escolares es mayor o se da por experimentación y, la ciudades de mayor riesgo de consumo de alguna vez en la vida fueron para México, Guadalajara y Las fronterizas de Tijuana y ciudad Juárez, (región noroccidental). Para Monterrey fue la más baja con $4,2 \%$, pero la prevalencia de uso en el último mes de $1,1 \%$ es tan alta como en la región norte ${ }^{(1)}$.

Para Nuevo León, la Encuesta nacional del consumo de drogas en la comunidad escolar reporta que el $8,2 \%$ de estudiantes de educación media había usado droga alguna vez en su vida, 4,4\% en el último año y 2,2\% en el último mes con tasas de prevalencia de 3,6\%;2,11\% y $0,75 \%$ respectivamente, para las drogas lícitas el consumo de alguna vez en la vida de $40,7 \%$ con prevalencia de tabaco y alcohol de $37,5 \%$ y $24 \%{ }^{(9)}$.

Las tasas de prevalencia de usuarios del área metropolitana en dos centros de tratamiento de drogas, registra alta prevalencia del uso de marihuana, incremento en el uso de cocaína, heroína y alucinógenos comportamiento similar al resto de centros de atención del país.

El promedio de edad de inicio para las susbtancias psicoactivas varia de los 12 y 13 años, para alcohol, tabaco y marihuana en población escolar de al igual que reportes internacionales e nacionales hay relación del consumo y problemas escolares, de conducta, uso indebido del tiempo libre y funcionamiento social disfuncional ${ }^{(9-11)}$.

Los estudios del consumo de drogas, lícitas e ilícitas, reflejan que cada vez más, son ofertada con menores restricciones a través de publicidad masiva y mensajes subliminales con representaciones sociales cotidianas de dominio, control, éxito, reconocimiento o sugiriéndola como una alternativa de satisfacción sin restricciones $^{(12-13)}$. Los grupos institucionales gubernamentales y no gubernamentales responden a esta propuesta con campañas preventivas también masivas, de carácter obligatorio y con rango de ley, pero los reportes de impacto o penetración no son optimistas ${ }^{(14)}$ y sugieren la necesidad de alternativas de menor costo y mayor efectividad.

Estudios de los factores de influencia de riesgo y protección de los individuos relacionados con el uso de drogas, refieren que la familia es la principal influencia en los primeros años pero que en la adolescencia son los grupos de la misma etapa. Consideran que las habilidades de comunicación y el establecimiento de normas en el hogar son recursos que se identifican como trascendentales para el fortalecimiento de la autoestima y capacidad de enfrentamiento a los problemas de la vida diaria, se les consideran como atributos valiosos que le ayudan a los individuos a tomar decisiones y resolver los problemas con asertividad ${ }^{(6,15)}$.

De las etapas de la vida la adolescencia representa una de las más críticas por su carácter transcicional y sometimiento, a las influencias sociales, internas y familiares que ejercen tanta presión en la 
satisfacción de sus necesidades básicas y de realización que ponen en riesgo su estabilidad emocional.

Los grupos de mayor riesgo de uso de drogas para el adolescente son los grupos delincuentes, las pandillas y mejores amigos con patrones de consumo de drogas lícitas e ilícitas ${ }^{(16-17)}$. Además de esa influencia se reconoce que existe la presión de los iguales como grupo de referencia más fuerte, que les proveen las bases de compromiso, negociación y socialización para confirmar su identidad, intimidad y conducta pública apropiada. La desaprobación de los amigos tiene un impacto mayor en el inicio del uso que la de los padres, y si esas actitudes están en conflicto con las de los iguales, ellos tienen más influencia $^{(18-19)}$.

Un estudio cualitativo sobre consumo de las drogas en adolescentes de diferentes culturas, se investigó las presiones a que eran sometidos los adolescentes para el uso de las mismas, concluyendo sus autores que estaban involucrados los siguientes conceptos: Patrón de consumo, Estrategias de resistencia y Perfil de consumo; en éstas últimas se incluyen las características conductuales, psicológicas y normativas de la persona, donde el patrón de consumo es la cantidad y frecuencia de ingesta de alcohol, tabaco u otras drogas en los últimos 30 días; las estrategias de resistencia, son las habilidades de resistencia usadas para evitar el uso de alcohol, tabaco $\mathrm{u}$ otras drogas, las cuales fueron categorizadas como: Rechaza - declinar una propuesta; Explica - dar una excusa o explicación y Levántate - abandono de una situación de ofrecimiento ${ }^{(20-21)}$.

\section{Definición de términos específicos}

Para facilitar la comprensión de términos que serán utilizados en esta investigación, en la secuencia se presenta la definición de los mismos según lo propone los especialistas del tema ${ }^{(12,20-21)}$.

Perfil de consumo: Son los rasgos de ingesta de alcohol, tabaco u otras drogas, según características demográficas, conductuales, psicológicas y de normas personales, normas preceptivas y, normas descriptivas, así; las características demográficas incluyen género, edad, religión, promedio escolar previo, relación con padres de los jóvenes así como la procedencia, ocupación y lugar de residencia de la familia. Las características conductuales, engloban la cantidad, frecuencia, edad de inicio, fuentes de información e involucramiento en compra- venta de drogas reciente o de toda la vida y las estrategias utilizadas para aceptar o rechazar el ofrecimiento. Finalmente, las características psicológicas, son la confianza percibida para decir no al ofrecimiento de familiares, amigos apreciados o poco conocidos de cualquier tipo de drogas. Incluye la autoeficacia, valoración de las intenciones y las expectativas o percepciones positivas asociadas por el uso de drogas.

La autoeficacia, es la confianza de decir no a propuestas de ofrecimiento de drogas a familiares o amigos apreciados o poco conocidos en cuanto las intenciones de uso se refieren a los propósitos que tienen para aceptar propuestas de drogas.

Las expectativas positivas de uso, representan las percepciones de las consecuencias positivas asociadas al uso de drogas como pasarla bien, ser parte del grupo, divertirse mejor o estar menos nervioso.

Las Normas pueden ser divididas en tres categorías: normas personales, las cuales se refieren a lo qué el individuo piensa que es correcto o equivocado del uso de alcohol, tabaco y otras drogas; Normas preceptivas, lo que el individuo cree que otros opinan del uso de alcohol, tabaco y otras drogas, (aquí se incluye la familia) y normas descriptivas, representando las creencias relacionadas a cuántos de sus amigos o compañeros usan drogas.

Los jóvenes de preparatoria del área metropolitana de Monterrey, Nuevo león, constituyen un grupo de riesgo para el consumo de drogas en el que es apropiado instituir, con determinación un programa preventivo, no obstante, conviene en primer lugar obtener un perfil de qué drogas han consumido recientemente; conocer cuáles son sus normas personales de resistencia a la posible oferta, si los amigos usan alguna droga y si en algún momento han sentido el impulso de aceptar algún ofrecimiento. Hecho que constituyó el propósito del presente estudio.

\section{MÉTODOS}

El diseño del estudio fue descriptivo, con un muestreo no probabilístico, de conveniencia en dos preparatorias de una universidad pública del Estado de Nuevo León, México, se seleccionaron 136 adolescentes en una y 179 en otra, para un total de 325 jóvenes de ambos sexos, que cursaban el primer o segundo semestre y tuviesen de 15 a 22 años de edad. Se usó la Encuesta Juvenil "Una Encuesta Sobre Mi Vida", desarrollada, por 
la Arizona State University (Drug Resistance Strategies Project) la cual reestructuraron a una versión para población mexicana, constando de 69 preguntas: 20 de las cuales, miden características demográficas, conductuales, psicológicas y normas para autoestima. La información se procesó en el paquete estadístico SPSS - 10.0, (Statistical Pakage for the Social Science, 2000), del cual se obtuvieron estadísticos descriptivos como frecuencias, porcentajes, medias y desviación estándar, de dispersión y pruebas de normalidad.

\section{RESULTADOS}

\section{Características demográficas}

El $56 \%$ son jóvenes del sexo masculino y $44 \%$ del femenino. El $79 \%$ dijo ser católico, el 12\% evangélico u otra religión, el resto refirió no creer ni practicar religión alguna, aunque $35 \%$ de ellos considera la religión importante. En cuanto a la asistencia a los servicios religiosos el $51 \%$ lo hace algunas veces o raramente y el $20 \%$ nunca lo hace.

Las edades de mayor predominio fueron las de 15 y 16 años que en conjunto representan el $88 \%$ del total de los jóvenes, y un $12 \%$ para los de 17 a 22 años.

El $91 \%$ de los jóvenes viven con ambos padres o con solo uno de ellos y el $9 \%$ con padrastros, familiares, compañeros o amigos. La ocupación de padre y madre es la de profesionales y pequeños empresarios en el $42 \%$ y el $20 \%$ de los casos respectivamente y $47 \%$ para el padre y $18 \%$ en el caso materno su trabajo es en áreas especializadas y no especializadas. La madre se dedica al hogar o es desempleada en el $62 \%$ de los casos. Así mismo el $97 \%$ de los jóvenes perciben la relación parental de muy buena a excelente si es con la madre y $78 \%$ si es con el padre. El $71 \%$ de los jóvenes reportan que su promedio escolar de secundaria fue de 80 a 100 puntos, y el $29 \%$ de 79 puntos; La meta escolar del $85 \%$ de ellos es terminar una carrera profesional o técnica mientras que solo un $15 \%$ aspira a terminar la preparatoria.

\section{Características conductuales}

En relación al uso de substancias psicotrópicas alguna vez en la vida, los datos sobre los encuestados, advierten que el 33\% ha consumido alcohol, el $35 \%$ ha fumado cigarros y el $93 \%$ ha fumado marihuana.
Aunado a lo anterior, el $67 \%$ relata haber bebido al menos una vez (de una hasta más de 50 bebidas), el $65 \%$ haber fumado una vez o más (de una fumada hasta más de 5 cajetillas) y el $7 \%$ que ha fumado marihuana una vez o más en su vida. Respecto al uso de otras drogas en la vida entre el 91 a 95\% de los jóvenes aseguró que ninguna vez las han usado y sólo el $5 \%$ o menos aceptó haberlo hecho una vez o más, incluso en el último mes. En la categoría ninguna vez en la vida el $67 \%$ de los jóvenes usaron bebidas alcohólicas, el $62 \%$ fumaron tabaco y el 97\% fumaron marihuana.

Sin embargo el porcentaje de la conducta de rechazo en los últimos treinta días de los jóvenes frente al ofrecimiento de drogas, muestra que al menos una vez 0 más rechazaron en un $56 \%$ el alcohol, $64 \%$ tabaco y $51 \%$ marihuana sin dar explicación.

Los jóvenes eventualmente evitan situaciones o lugares en que puede darse el ofrecimiento como una medida de rechazo, la frecuencia en que esto sucedió con la muestra del estudio fue de evitación, siendo más frecuente para el alcohol (49\%) que para el tabaco (39\%) pero superadas ambas con un $54 \%$ en el caso de la marihuana.

La aceptación de los jóvenes a ofrecimientos recientes (últimos treinta días) indica que en el caso de las tres substancias, al comparar la aceptación de una vez o más con el rechazo, el margen es de tan solo $15 \mathrm{y}$ $22 \%$ para el alcohol y tabaco en contraste con el $64 \%$ de la marihuana.

\section{Características psicológicas}

Esta variable buscó conocer la confianza con la que el joven resiste el ofrecimiento y sus intenciones de aceptar las substancias, ese dato se muestra en la Figura 1.

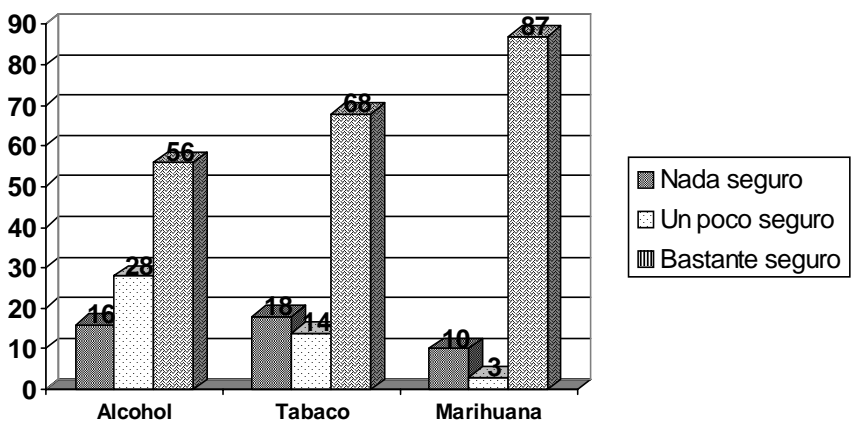

Fuente: ESV, 2003 $n=325$

Figura 1 - Confianza de resistir el ofrecimiento de drogas 
Los jóvenes reportaron estar bastante seguros de resistir a la oferta de marihuana (97\%) y para el alcohol y tabaco con un $56 \%$ y $68 \%$ respectivamente, en conjunto un $34 \%$ aceptaron no estar seguros de resistirse a las tres substancias.

Este comportamiento se complementa con el informado en las intenciones de aceptar el ofrecimiento, tal como es descrito en la Figura 2.

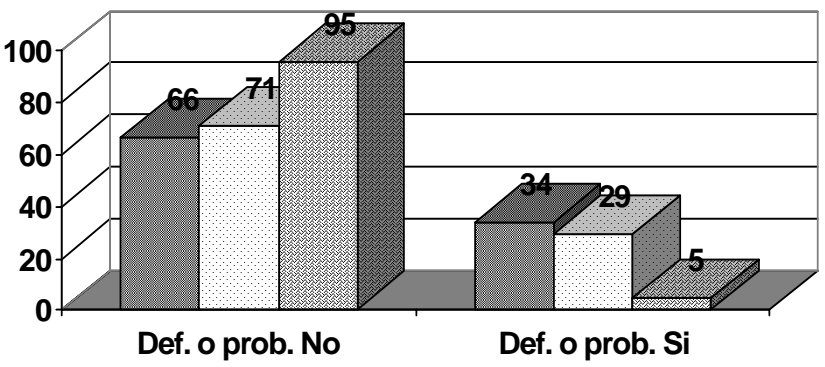

$\square$ Alcohol $\square$ Tabaco 图 Marihuana

Fuente: ESV, 2003

$$
\mathrm{n}=325
$$

Figura 2 - Intenciones de los jóvenes ante el ofrecimiento de alcohol, tabaco o marihuana en fin de semana

Esta figura refleja que es mayor la intención definitiva o probable de no aceptar la marihuana en el $95 \%$ que para el tabaco y alcohol con 71 y $66 \%$, respectivamente. Pero es mayor la proporción de los muchachos que tienen intención de aceptar el alcohol y tabaco (63\% acumulado) que el $5 \%$ referido para la marihuana. Los efectos positivos que los jóvenes asocian al usar alcohol o tabaco, son representados en la Figura 3.

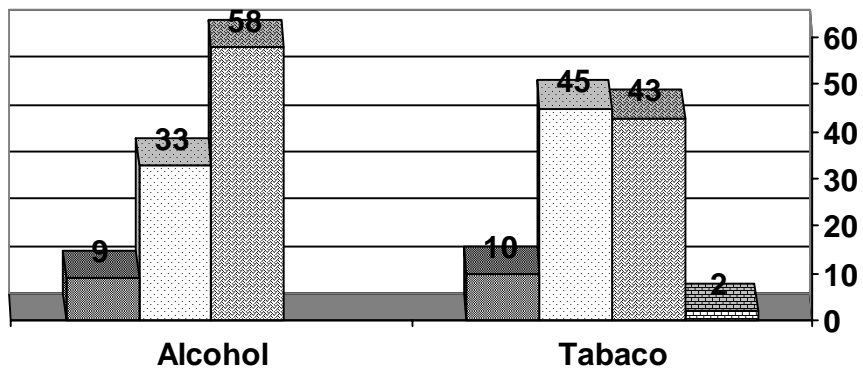

Casi siempre Con frecuencia 圆 Nunca 葍 Sin respuesta

Fuente: ESV, 2003 $\mathrm{n}=325$

Figura 3 - Expectativas positivas de uso de alcohol y tabaco

En esta figura se observa que si bien el 58 y $43 \%$ no le dan connotación positiva al uso de alcohol y tabaco, al acumular el porcentaje de frecuente y casi siempre (42 y $55 \%$ respectivamente), representan casi la mitad que mantiene expectativas positivas al uso de esas substancias.

Normas personales

En cuanto a normas personales, en su mayoría los jóvenes externaron desacuerdo si otros usan alcohol, tabaco y marihuana. Llama la atención que $27 \%$ dijeron lo contrario, esto se aprecia objetivamente en la siguiente Figura 4.

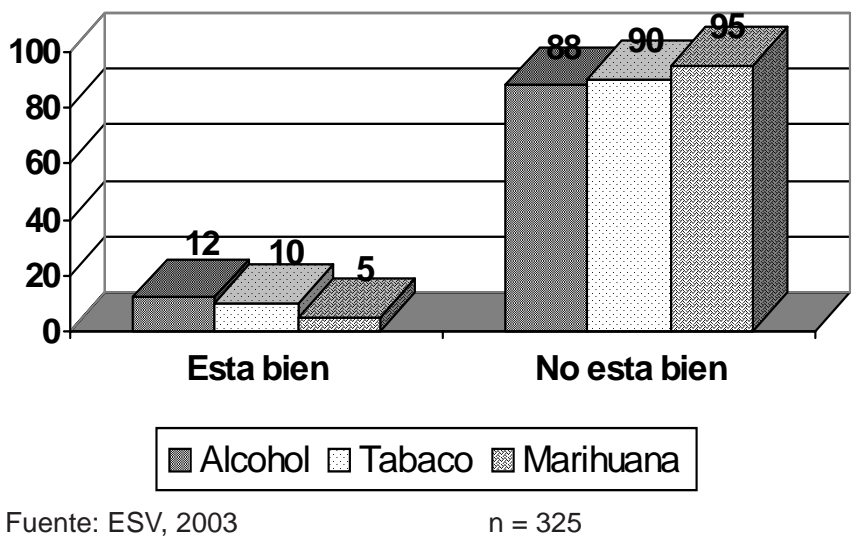

Figura 4 - Punto de vista de los jóvenes si alguien de su edad consume alcohol, tabaco o marihuana

Los jóvenes consideraron que sus padres aún cuando reaccionen con bastante enojo, este seria mayor tratándose de marihuana (98\%) que para el alcohol (81\%). En el caso de los amigos, la reacción de reprobación es mayor para la marihuana con $78 \%$ que para el alcohol y tabaco, con 27 y $28 \%$ cada uno respectivamente.

El $65 \%$ de los jóvenes advirtieron que la mayoría de la población escolar ha probado drogas y $48 \%$ de ella la usa regularmente.

Respecto al uso de drogas por los amigos, el $25 \%$ de los jóvenes consideran que ninguno de sus amigos lo hacen y el $75 \%$ que varios de sus amigos lo hacen, al menos una vez por mes.

En cuanto a la autoestima de los jóvenes, se elaboró un índice, el cual mostró una media bastante baja de 51.42 una D.E de 14.36 con un valor $D=\mathbf{3 . 1 4 3}$ y una $p$ $\mathbf{0} \mathbf{0 . 0 0}$, respecto a las características conductuales y normas de los estudiantes.

\section{DISCUSIÓN}

Según los datos de la encuesta nacional de 
adicciones $^{(1)}$, entre la comunidad escolar, el $8.2 \%$ de los estudiantes de educación media había usado droga alguna vez en su vida, y $2.2 \%$ en el último mes. En este estudio, se rebasa ampliamente este dato, dado que los preparatorianos aceptan haber usado alcohol, tabaco o marihuana alguna vez en su vida en un $67 \%, 65 \%$ y $7 \%$ respectivamente; y en el último mes en un $33 \%, 38 \%$, y $3 \%$ respectivamente para cada substancias.

La información reportada por el consejo nacional de adicciones $^{(1)}$, se ratifica entre los estudiantes de esta investigación, donde predomina el uso de tabaco y alcohol aunque en porcentaje menor, también consuman marihuana.

Aceptando las declaraciones de Wilks et $\mathrm{al}^{(16)} \mathrm{y}$ Morgan \& Grubbe ${ }^{(17)}$ los grupos de mayor riesgo para uso de drogas por el adolescente, entre otras influencias, son los mejores amigos con patrones de consumo de drogas lícitas e ilícitas; y las apreciaciones de Rawlins ${ }^{(18)}$ y Doyvan \& Adelson ${ }^{(19)}$, en el sentido de que son ellos los que les proveen de aportes para el ingreso en la maturidad; los jóvenes de esta investigación estarían ante dicho riesgo porque el $65 \%$ considera que la mayoría de sus compañeros han probado alcohol, tabaco u otras drogas, $16 \%$ que la mitad lo ha hecho y $19 \%$ que algunos han probado estas sustancias, así mismo que tienen al menos seis amigos o más que usan. Se registra un porcentaje mínimo de jóvenes que consideran que sus amigos les reprobarían la conducta de uso de sustancias $(27 \%$ alcohol, $28 \%$ tabaco y $78 \%$ marihuana).

No obstante el posible rechazo de amigos, al consumo de tabaco (62\%) y alcohol (67\%) reportado por los estudiantes de esta investigación, es mayor al encontrado por Jhonston, O'malley \& Bachman ${ }^{(3)}$ y Watts \& Wright $(1990)^{(4)}$ en población estadounidense (22.4\% y $31.5 \%$ respectivamente) del primer año de preparatoria. Pero menor al reportado en centros escolares por Branch $^{(14)}$, donde el tabaco es consumido por el $88 \%$, alcohol el $42 \%$ y por el $74 \%$ la marihuana.

El estudio permitió evidenciar los conceptos descritos por Marsiglia et al ${ }^{(20)}$, Harthun et al $^{(21)}$ acerca del patrón de consumo de drogas en adolescentes, en especial en las estrategias de resistencia, donde los estudiantes argumentaron que en un $56 \%$ tuvieron una vez o más que rechazar ofrecimiento de alcohol, de tabaco un $64 \%$ y de marihuana un $51 \%$ en el último mes, en la mayoría de los casos sin dar explicación, otra forma similar o bien alejándose de quien ofrece la sustancia. Esta variable de estrategias de resistencia, mostró una media de 33.53 entre los jóvenes.

En cuanto al perfil de consumo, entre los sujetos de estudio, la gran mayoría residente en la zona urbana y del sexo masculino, se muestra una media general de cantidad y frecuencia de 26.53 y D.E de 23.92.

Las características psicológicas que establecen los autores referidos en el estudio, implican en indagar sobre la confianza que en sí misma tiene la persona para resistir el ofrecimiento de drogas, sobre este punto, los jóvenes muestran inseguridad en un $28 \%$ para resistir la oferta de alcohol, $16 \%$ para la de tabaco y $10 \%$ la de marihuana. El 95\% afirma que definitivamente tiene la intención de negarse a la marihuana, y en un $74 \%$ y $66 \%$ respectivamente al alcohol y al tabaco. Esta variable muestra una media alta (69.03) respecto a las intenciones (22.92) y ligeramente menor frente a las expectativas positivas de uso (70.12).

Las normas preceptivas para el consumo que señalan los autores ${ }^{(20-21)}$, muestran alto el porcentaje en cuanto a que el enojo paterno se haría evidente si ellos consumieran drogas. Por cuanto a las normas descriptivas, la mayoría de los amigos reprueba el uso de drogas (cocaína, crack, LSD, inhalantes y alcohol).

Los resultados presentados y su coincidencia con la literatura refuerza la sugerencia de algunos autores ${ }^{(22)}$, en el sentido de que debe reforzarse el sentimiento de autoeficacia (confianza para rechazar) y el manejo de la presión social para el consumo de drogas, por cuanto hay compañeros de los jóvenes, muy cercanos que las usan.

\section{CONCLUSIONES}

En la presente investigación fue posible evidenciar los conceptos sobre el patrón de consumo de drogas, entre la muestra de estudiantes.

Se constató que la mayoría de los jóvenes son de estrato urbano y viven con sus padres y también, que han estado en la alternativa de aceptar o no ofrecimiento de alcohol, tabaco o marihuana a la cual han respondido con estrategia de rechazo al ofrecimiento al alcohol, tabaco 0 marihuana (alrededor del 50\% de los entrevistados) mediante explicación, alejarse o bien de otras formas.

Los jóvenes en un 31\%, 36\% y $13 \%$ han aceptado en los últimos 30 días alcohol, tabaco y marihuana respectivamente.

Aunque los datos hayan indicado puntajes de 
aceptación considerables de alcohol y tabaco en el último mes (promedio de 30\%) y un uso en la vida para esas substancias bastante alto (promedio de $65 \%$ ).

Como factor adicional se observa la exposición de los adolescentes a las drogas (lícitas e ilícitas), bien como a una comodidad estudiantil que posee un índice de aprobación al uso de drogas numéricamente considerable (70\%), y en que $50 \%$ de los jóvenes afirma que la mayoría de sus compañeros han probado esas substancias, con el agravante de que tienen amigos próximos que los han usado.

Los resultados caracterizan a ese grupo como de riesgo justificando la inversión en programas preventivos orientados a apoyar los recursos del adolescente para resistir las presiones relacionadas con el consumo de alcohol, tabaco y otras drogas.

El instrumento "Encuesta sobre mi vida" con adolescentes, dado su consistencia interna, (incluyendo las adecuaciones para la población mexicana) fue un recurso metodológico importante para el desarrollo del

\section{REFERENCIAS BIBLIOGRÁFICAS}

1. Centro de Investigación Juvenil AC (MX). Encuesta de adicciones. [Serie en Rede]. 1998. Disponible en: URL: ssa.gob.mx/unidades/conadic/CDM. Htm

2. Instituto Mexicano de Psiquiatria. Diagnostico y tendencias del uso de drogas en México: Secretaria de Salud; 2002.

3. Johnston LD, O'Malley PM, Bachman JG. National trends in drug use and related factors among American high school students and young adults, 1975-1986. Washington (DC): U.S. Government Printing Office; 1998.

4. Watts WD, Wright LL. The relationship of alcohol, tabacco, marijuana, and other illegal drug use to delinquency among Mexican-American, black and white adolescent males. Adolescence 1990; 25:171-81.

5. De Micheli D, Formigoni ML. As razões para o primeirouso de drogas e as circunstancias familiares prevem os padrões de uso futuro. J Bras Dependência Químicas 2001 Set; 2(1). 6. Galduroz JCF, Noto AR, Carlini EA. IV Levantamento sobre uso de drogas entre estudantes de $1^{\circ}$ e $2^{\circ}$ graus em 10 Capitais Brasileira. São Paulo (SP): CEBRID Centro Brasileiro de informações sobre drogas psicotropicas Dep. de Psicologia da Escola Paulista de Medicina da Universidade Federal de São Paulo; 1997.

7. Frank TB, Humberto JB, Silva LM. Prevalencia do uso de drogas e desempenho escolar entre adolescentes. Rev Saúde Pública 2001; 35(2):150-8.

8. Conadic - Consejo Nacional de las Adicciones Encuestas. Diagnostico y tendencias del uso de drogas en México. México: Secretaria de Salud; 1999.

9. Arrellanez HJ, Díaz ND. Prevalencia del Uso de Drogas y Factores Psicosociales Asociados entre Estudiantes de Educación Media Básica del Estado de Nueva León (PA 01). Nuevo León: Centro de Integración Juvenil; 2001. estudio y con potencial de aplicación para futuras investigaciones.

Como los resultados han indicado la necesidad se pretende complementar el presente estudio con una intervención implementando en los planteles educativos, el programa "Mantente REAL" de prevención del uso de drogas de la Arizona State University.

\section{AGRADECIMIENTOS}

Agradecemos a todos los docentes de la Escuela de Enfermería de Ribeirão Preto y a los treinta y tres participantes de nueve países latinoamericanos que participaron en el proceso de implementación de los programas, y también al Gobierno del Japón y al Programa de Becas de la OEA por su apoyo financiero y becas que posibilitaron la implementación del "I Programa Regional de Capacitación en Investigación para Enfermeros de América Latina".

10. Mora RJ, Natera G. Expectativas, consumo de alcohol y problemas asociados en estudiantes universitarios de la ciudad de México. Salud Pública México 2001 mzo-abr; 43(2). 11. Alvarez J. Representaciones e Intervención: Los jóvenes entre el alcohol. México: Universidad Autónoma de Nuevo León; 1997.

12. Bucher R, Landini TM. Conhecimento e uso de drogas entre alunos de Brasília. Psic Teor e Pesqui; 3(2):178-94.

13. Willie A, Holibar, CS, Fuamutu N, Aiolupatea, K, MoewakaB, Aroha P. A qualitative investigation of responses to televised alcohol advertisements. Contemporary drug Problems 24/ Spring. 1997

14. Luis AVM, Pillon SC, Costa LA, Oliveira PP. O que os estudantes captam nas propagandas sobre álcool e drogas: um estudo piloto..inédito. Ribeirão Preto (SP): EERP; 1999. 15. Velásquez Hernández ML. Factores de riesgo y etapas de adquisición del uso de drogas en estudiantes de bachillerato. 2002.

16. Morgan M, Grube JW Closeness and peer group influence. Br J Soc Psychol 1991; 30:159-69.

17. Wilks J, Callan VJ, Austin DA. Parent, peer and personal determinants of adolescent drinking. $\mathrm{Br} \mathrm{J}$ Addiction 1989; 84:619-30.

18. Rawlins WK. Friendship matters. New York: Aldine De Gryter; 1992.

19. Doyvan E, Adelson J. The adolescent experience. New York: Wiley; 1996.

20. Marsiglia FF, Kulis S, Hecht ML. Ethnic labels and ethnic identity as predictors of drug use and drug exposure among middle school students in the Southwest. J Res Adolescence 2001; 11(1): 21-48.

21. Harthun ML, Drapeau AE, Dustman PA, Marsiglia FF. Implementing a prevention curriculum: An effective researcherteacher partnership. Educ Urban Soc 2002; 34(3):353-64. 22. Miller MA, Alberts JK, Hecht ML, Trost MR, Krizek RL. Adolescent relationship and drug use. Ed. LEA; 1998. 